\title{
Measurement and modeling of the effective thermal conductivity for porous methane hydrate samples
}

\author{
LI DongLiang ${ }^{1,2,3}$, DU JianWei ${ }^{1,2,3}$, HE Song ${ }^{1,2,3}$, LIANG DeQing ${ }^{1,2 *}$, \\ ZHAO Xiang Yong ${ }^{1,2}$ \& YANG XiangYang ${ }^{1,2}$ \\ ${ }^{1}$ Key Laboratory of Renewable Energy and Gas Hydrate, Guangzhou Institute of Energy Conversion, Chinese Academy of Sciences, \\ Guangzhou 510640, China \\ ${ }^{2}$ Guangzhou Center for Gas Hydrate Research, Chinese Academy of Sciences, Guangzhou 510640, China \\ ${ }^{3}$ Graduate School of the Chinese Academy of Sciences, Beijing 100039, China
}

Received January 23, 2011; accepted May 4, 2011; published online December 2, 2011

\begin{abstract}
The effective thermal conductivities of gas-saturated porous methane hydrates were measured by a single-sided transient plane source (TPS) technique and simulated by a generalized fractal model of porous media that based on self-similarity. The density of porous hydrate, measured by the volume of the sample in the experimental system, was used to evaluate the porosity of methane hydrate samples. The fractal model was based on Sierpinski carpet, a thermal-electrical analogy technique and one-dimensional heat flow assumption. Both the experimental and computational results show the effective thermal conductivity of methane hydrate decreases with the porosity increase. The porosity of 0.3 can reduce the thermal conductivity of the methane hydrate by $25 \%$. By analysis of the experimental data and the simulative result, the optimized thermal conductivity of the zero-porosity methane hydrate is about $0.7 \mathrm{~W} \mathrm{~m}^{-1} \mathrm{~K}^{-1}$.
\end{abstract}

gas hydrate, thermal conductivity, fractal geometry model, porosity

\section{Introduction}

Large natural gas (mainly methane) hydrate deposits are found worldwide in sediments of outer continental margins and polar permafrost areas [1]. It has been estimated the combustible carbon in naturally occurring methane hydrates is at least twice as much as that in all other fossil fuels on the Earth. Gas hydrates are believed as a potential future energy source and their thermal properties play an important role in assessing gas production from natural deposits, sea floor stability of hydrate-bearing oceanic sediments, global climate change, submarine slide formation, and hydrate plug dissociation in oil and gas pipelines [2-4]. Thermal conductivity measurements, therefore, are crucial in quantifying the role of gas hydrates in these applications. Howev-

*Corresponding author (email: Liangdq@msl.giec.ac.cn) er, few measurements are available in literature and the limited measurement data are largely different.

A zero-porosity sample is a key to determine hydrate thermal conductivity, but it is difficult to get a sample without free water and free gas in laboratory. Especially, as the hydrate sample formed from ice powders, unconverted ice sometimes remains in the center surrounded by gas hydrates. Cryo-SEM studies of Kuhs et al. [5] showed the hydrates were composed of spongelike networks with 40-400 $\mathrm{nm}$ diameter gas-filled pores. The pores may occupy $15 \%-20 \%$ of the hydrate volume. The elliptical and branched pore networks were closed and inaccessible to water for further reaction; some channels with diameters of a few $\mu \mathrm{m}$ formed in the hydrate matrix. Different void fraction and fluids in void space of hydrate result in the variation of effective thermal conductivity. The experiments of Rosenbaum [6] showed that the thermal conductivity ranged from 0.264 to $0.338 \mathrm{~W} \mathrm{mK}^{-1}$ corresponding to a void fraction of 
about 0.10 to 0.25 for methane hydrates. deMartin [7] reported that the thermal conductivity of a porous structure I methane hydrate sample was $0.32 \pm 0.005 \mathrm{~W} \mathrm{~m}^{-1} \mathrm{~K}^{-1}$ at 263 $\mathrm{K}$ and a confining pressure of about $28 \mathrm{MPa}$. The methane hydrate samples measured by deMartin were estimated to have a porosity of $28 \%$ to $33 \%$, determined by knowing the volumes of the hydrate sample and the vessel. Huang and Fan [2] reported that the thermal conductivity of the methane hydrate formed from SDS solution increased from 0.33 to $0.57 \mathrm{~W} \mathrm{~m}^{-1} \mathrm{~K}^{-1}$ with $2 \mathrm{MPa}$ impaction in the axial direction, but the porosity and density data information were unaccounted. Besides the experimental measurements, many models to predict the effective thermal conductivity of porous medium, either empirical or theoretically based but specific to a given material, have been proposed. However, few inverse models of the effective thermal conductivity are available in the literature for porous methane hydrate. Gupta et al. [8] compared the mixing models of the arithmetic, harmonic, geometric, and square root mean models, respectively, to independently predict the composite thermal conductivity of porous methane hydrate as a function of phase saturation. Hence, it is necessary to study the effective thermal conductivity of porous methane, especially the zero-porosity methane hydrate thermal conductivity via model calculation.

In this work, we determined the effective thermal conductivity of synthetic porous hydrate samples by a single-sided transient plane source (TPS) technique and molding the effective thermal conductivity by a generalized model of porous media based on self-similarity.

\section{Experiment}

\subsection{Apparatus}

The measurement apparatus includes a hydrate formation system and a thermal conductivity test system, as shown in Figure 1. This device permits the formation and subsequent direct compaction of the sample on a TPS element attached to an insulating support. The hydrate formation system includes a gas reaction vessel, an air-cooling bath, a gas support unit, a sample compacted unit and a data recording unit.

More details of the cylindrical stainless steel pressure vessel are shown in Figure 2. The inside diameter is $5.0 \mathrm{~cm}$ and the sample can be compacted by a hydraulic piston that is promoted by hydraulic pressure with the outside hand pump. A liner motion sensor, fixed on the side of the vessel, is used to measure the length of the sample. The whole vessel is placed inside an Angelantoni Challenge 250 climatic chamber that provides temperature control within $0.1 \mathrm{~K}$ and can be programmed for temperature ramp cycles and soak intervals. The temperature of the sample cell is measured by a PT100 with an accuracy of $\pm 0.01 \mathrm{~K}$ and the pressure is measured by a pressure sensor with an accuracy of $\pm 20 \mathrm{kPa}$. Data from the gas pressure, the temperature and the piston motion are collected by an Agilent 34970 Data Acquisition/Switch Unit and logged by an Agilent BenchLink Data Logger program.

The thermal conductivity of the sample is measured by the Hot Disk Thermal Constants Analyser system, which consists of the Hot Disk sensor, bridge, keithley 2000 multimeter, keithley 2400 multimeter, and computation device. The Hot Disk probe is made of a $10 \mu \mathrm{m}$ thick Nickel-metal double spiral. The contact parts and the probe are protected inside the polyimide shell that is no more than $0.5 \mathrm{~mm}$ thick. The outer $\varphi 66 \mathrm{~mm}$ foil, which protects the probe from damage and supports the soft probe at the same time, is glued to the central spiral part. The sensor acts both as a heat source for increasing the temperature of the sample and as a resistance thermometer for recording the time-dependent temperature increase. To allow a sample to be compacted in situ, a one-sided TPS technique is utilized by adhering the TPS to polytetrafluoroethylene (PTFE), which serves as a backing support and partial insulator.

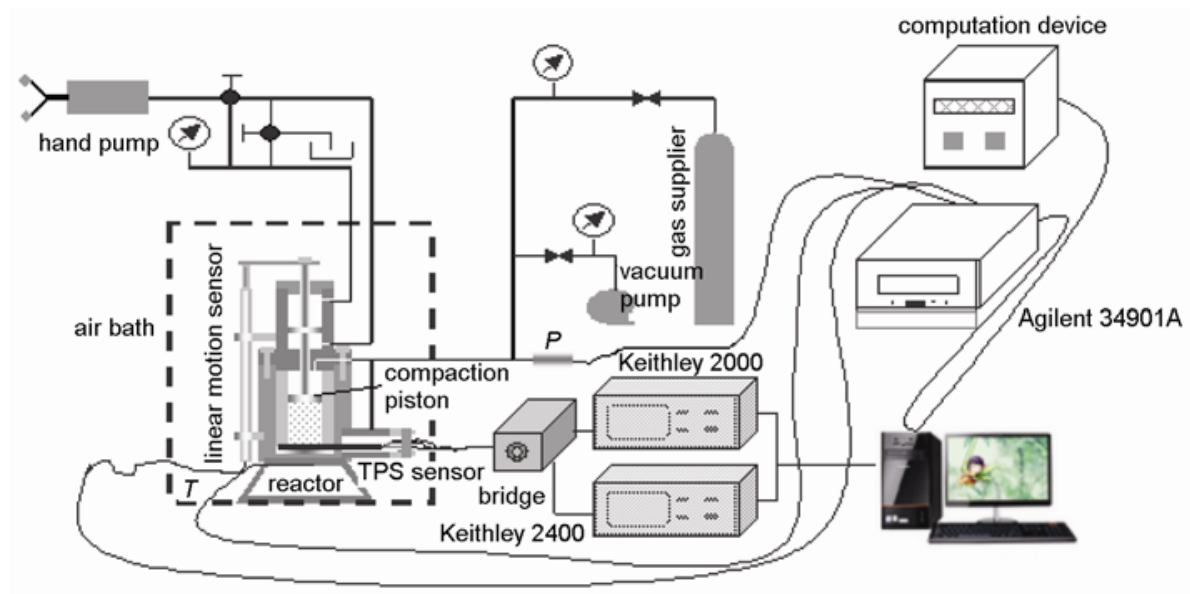

Figure 1 The schematics of methane hydrate formation and thermal conductivity measurement system. 


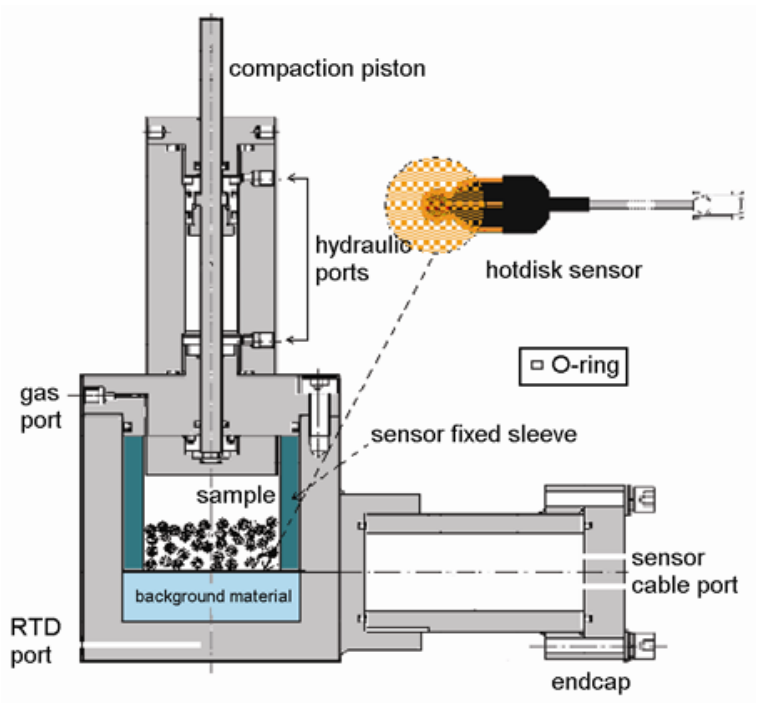

Figure 2 Reactor of in situ measurement of gas hydrates thermal conductivity.

\subsection{Procedure}

It's found surfactants, such as sodium dodecyl sulfate (SDS), could increase gas hydrate formation rate and reduce the formation time and make it easier to get a porous methane sample [2]. In this work, the methane hydrate sample for thermal conductivity test was formed from SDS solution instead of distilled water.

Prior to the test, the reactor was rinsed with double distilled water and then dried by a blower. To control the temperature during the sample synthesis and thermal conductivity measurements, the reactor was placed in a temperature controlled air bath after sealing. Then, the reactor was vacuumed and the $300 \mathrm{ppm}$ SDS aqueous solution with a weight of about $30 \mathrm{~g}$ was carefully imbibed by the negative pressure ensuring the height of the sample was about $15 \mathrm{~mm}$, which was greater than the probing depth calculated according to Gustafsson's equation for probing depth. Subsequently, the pore pressure within the sample was raised to $16 \mathrm{MPa}$ and the temperature of the chamber was cycled from 277 to $258 \mathrm{~K}$ with $1 \mathrm{Kh}^{-1}$. This was repeated until no further gas uptake (pressure decrease) or ice formation (pressure increase) from unreacted water was evident from the pressure data. The hydrate sample was then compacted with mechanical pressure by hydraulically pushing the internal piston of the pressure vessel, which moved down to contact and compact the sample. The compaction pressure was increased step by step. At the same time, the effective thermal conductivity was measured under each compaction pressure.

\section{Modeling and programming}

Because the Euclidean geometry has a limitation in de- scribing the structure of pores, traditional treatment of porous media has to employ the continuous medium assumption and the volumetric averaging method, which make it difficult to consider the influence of microstructures of pores, and thus the application of these existing porous media theories have some fundamental limitations. However, fractal theory may provide a new method to study the effective thermal conductivity of porous media. Thovert $e t$ al. [9] and Adler [10] have applied a Sierpinski carpet model to simulate real porous media and note that the porous media are random and deterministic fractals. Ma et al. [11] used the Sierpinski carpet as a geometry model and developed a self-similarity model to simulate the statistically self-similar porous medium to predict the thermal conductivity of twophase/saturated porous media based on a thermal-electrical analogy technique and one dimensional heat flow assumption. In addition, some researchers have proven that gas hydrates are fractals statistically, meaning that they are statistically self-similar $[12,13]$. As a result, we can use the fractal geometry model to study the effective thermal conductivity of heterogeneous porous methane hydrate samples and calculate the thermal conductivity of zero-porosity methane hydrate.

Sierpinski carpets are a special class of two-dimensional fractals and they have been proposed as a model for constructing porous and fractured media [14]. Sierpinski carpets can be constructed with a given geometric generator. In Figure 3, the generator is a square divided into $L_{0} \times L_{0}$ (here, $L_{0}$ is 3.) congruent sub-squares, where $C_{0}^{2}$ is black and the other $L_{0}^{2}-C_{0}^{2}$ is white. The black sub-squares remain, whereas the white ones will be removed. In the next iteration stage, each of the remaining sub-squares is again subdivided into $L_{1} \times L_{1}$ equal sub-squares and the pattern of the generator is repeated. An iterative repetition of these steps then creates a structure with pores and matrix solids of various

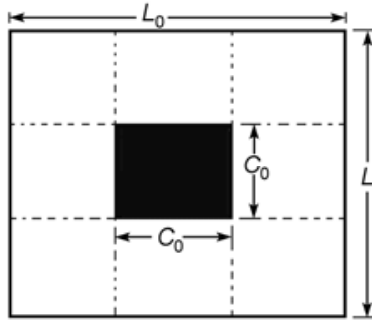

(a)

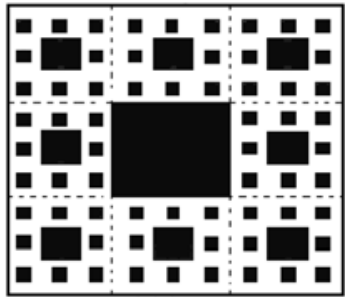

(c)

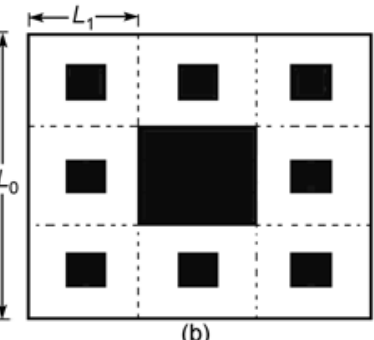

(d)

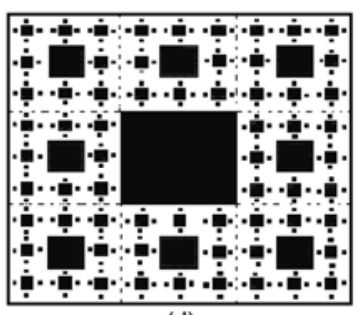

Figure 3 Sierpinski carpets of different stage. (a) 0-Stage; (b) 1-stage; (c) 2-stage; (d) 3-stage. 
sizes.

This construction procedure repeated infinitum can generate a series of self-similar Sierpinski carpets. Because Sierpinski carpet is a kind of two-dimensional fractal, the fractal dimension is given by:

$$
D_{\mathrm{f}}=\frac{\ln \left(L^{2}-C^{2}\right)}{\ln (L)}
$$

If $L=3$ and $C=1$ (Figure 3), the fractal dimension $D_{\mathrm{f}}$ is 1.893. All the $n$-stage $(n=0,1,2,3, \ldots)$ have the same structure and the same fractal dimension. Hence, the porosity in this work is given by:

$$
\phi=\left(\frac{L^{2}-C^{2}}{L^{2}}\right)^{n+1}=\left(\frac{8}{9}\right)^{n+1}
$$

where the superscript $n$ represents the stage of the Sierpinski carpet and $n=0,1,2 \ldots$.

Three assumptions or approximations are made for this study: (1) The porous medium consists of two portions (randomly distributed non-touching particles and self-similarly distributed particles contacting each other with thermal resistance between them); (2) one-dimensional heat flow across porous, more or less isotropic saturated sand with no large distortion of heat flow $[15,16]$; (3) conductive heat resistance is analogous to electrical resistance. The heat conduction model and parallel electrical analogues of the thermal resistances for 0-stage Sierpinski carpet are shown in Figure 4. Here, the bar represents the contact resistance between the solid particles depending on the bar width $t$. In this model, $t=0$ means no contact between particles. Since the Sierpinski carpet is a self-similar fractal geometry, the contact thermal resistances (bar widths) are also assumed to be self-similar. To simplify the calculation, we used the dimensionless parameter $t^{+}(=t / L)$ and took it to be constant. Ma et al. [11] proved that the lateral contact resistance is not important and the one-dimensional heat flow assumption at steady state is applicable.

Figure 4(b) shows the electrical analogy diagram for the 0 -stage Sierpinski carpet, where only the contact resistances along the heat flow direction are considered. If the thermal conductivity of fluid is $k_{\mathrm{f}}$ and the thermal conductivity of porous media frame is $k_{\mathrm{m}}$, thermal resistance of layer 1 is considered to be the parallel equivalent for $R_{11}, R_{12}, R_{13}$, and it is:

$$
R_{1}^{(0)}=\frac{R_{1} R_{1 \mathrm{~b}}^{(0)}}{R_{1}+R_{1 \mathrm{~b}}^{(0)}}=\frac{L^{(0)}-C^{(0)}}{2 L^{(0)} k_{\mathrm{f}} L^{(0)}\left[t^{+} \beta^{(0)}+\left(1-t^{+}\right)\right]}
$$

where, $\beta^{(0)}=k_{\mathrm{m}} / k_{\mathrm{f}}$.

The thermal resistance of layer 2 is:

$$
\begin{aligned}
R_{2}^{(0)} & =\frac{R_{21}^{(0)} R_{22}^{(0)} R_{23}^{(0)}}{R_{22}^{(0)} R_{23}^{(0)}+R_{21}^{(0)} R_{23}^{(0)}+R_{21}^{(0)} R_{22}^{(0)}} \\
& =\frac{C^{(0)}}{k_{\mathrm{f}}\left[L^{(0)}-C^{(0)}+\beta^{(0)} C^{(0)}\right]}
\end{aligned}
$$

Similarly, the thermal resistance of layer 3 is:

$$
R_{3}^{(0)}=\frac{R_{3} R_{3 \mathrm{~b}}^{(0)}}{R_{3}+R_{3 \mathrm{~b}}^{(0)}}=R_{1}^{(0)}
$$

The total resistance of the 0 -stage Sierpinski carpet is considered as the series equivalent of layers 1,2 and 3, and it is:

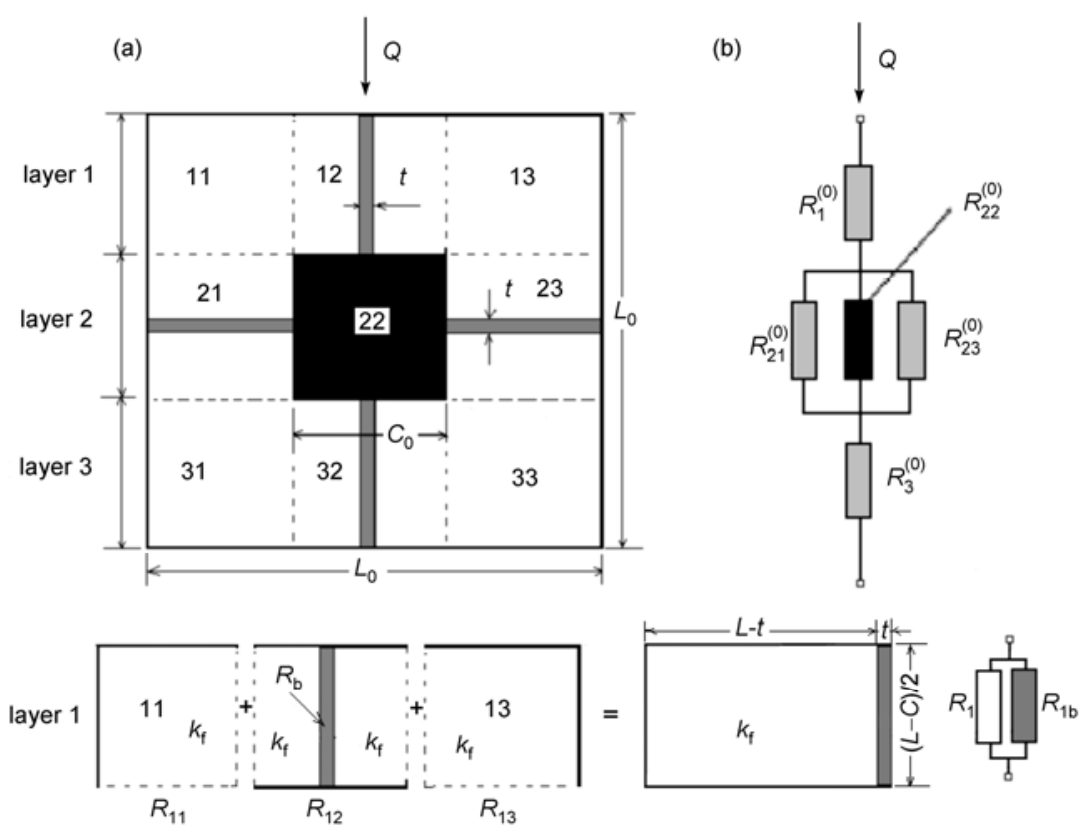

Figure 4 The thermal conductivity model and the thermal-electrical analogy for a 0-stage Sierpinski carpet. (a) The thermal conductivity model for a 0 -stage carpet; (b) the network of the thermal-electrical analogy for a 0-stage carpet. 


$$
\begin{aligned}
R_{\mathrm{SC}}^{(0)} & =R_{1}^{0}+R_{2}^{0}+R_{3}^{0} \\
& =\frac{1}{k_{\mathrm{f}}}\left(\frac{1-C^{(0)} / L^{(0)}}{t^{+}\left(\beta^{(0)}-1\right)+1}+\frac{C^{(0)}}{C^{(0)}\left(\beta^{(0)}-1\right)+L^{(0)}}\right)
\end{aligned}
$$

Thus, the dimensionless effective thermal conductivity for the 0 -stage carpet can be obtained by:

$$
\begin{aligned}
k_{\mathrm{e}, \mathrm{SC}}^{+(0)} & =\frac{k_{\mathrm{e}, \mathrm{SC}}^{(0)}}{k_{\mathrm{m}}}=\frac{L_{0}}{R_{\mathrm{SC}}^{(0)} A_{0} k_{\mathrm{m}}} \\
& =\left(\frac{1-C^{(0)} / L^{(0)}}{t^{+}\left(\beta^{(0)}-1\right)+1}+\frac{C^{(0)}}{C^{(0)}\left(\beta^{(0)}-1\right)+L^{(0)}}\right)^{-1}
\end{aligned}
$$

where, $A_{0}=L_{0} \times 1$.

A similar method can be used to derive the effective thermal conductivity of a 1-stage carpet. In Figure 3(b), except for the central shaded region with conductivity $k_{\mathrm{s}}$, the other regions are considered as an equivalent homogeneous material with thermal conductivity $k_{\mathrm{e}, \mathrm{SC}}^{(0)}$. Thus, the dimensionless effective thermal conductivity of the 1-stage carpet is:

$$
k_{\mathrm{e}, \mathrm{SC}}^{+(1)}=k_{\mathrm{e}, \mathrm{SC}}^{+(0)} \times\left(\frac{1-C^{(1)} / L^{(1)}}{t^{+}\left(\beta^{(1)}-1\right)+1}+\frac{C^{(1)}}{C^{(1)}\left(\beta^{(1)}-1\right)+L^{(1)}}\right)^{-1}
$$

where, $\beta^{(1)}=\beta^{(0)} / k_{\mathrm{e}, \mathrm{SC}}^{+(0)}$.

Similarly, the dimensionless effective thermal conductivity for an $n$-stage carpet can be given by:

$$
k_{\mathrm{e}, \mathrm{SC}}^{+(n)}=k_{\mathrm{e}, \mathrm{SC}}^{+(n-1)} \times\left(\frac{1-C^{(n)} / L^{(n)}}{t^{+}\left(\beta^{(n)}-1\right)+1}+\frac{C^{(n)}}{C^{(n)}\left(\beta^{(n)}-1\right)+L^{(n)}}\right)^{-1}
$$

where, $\beta^{(n)}=\beta^{(0)} / k_{\mathrm{e}, \mathrm{SC}}^{+(n-1)}, n=1,2, \cdots$.

Eq. (9) presents the recursive algorithm for the thermal conductivity of self-similar porous media.

In this model, it is assumed that the porous medium consists of two portions and the two portions are considered to be in parallel. So, the total thermal resistance can be expressed as:

$$
\frac{1}{R_{\mathrm{t}}}=\frac{1}{R_{\mathrm{nt}}}+\frac{1}{R_{\mathrm{SC}}}
$$

The effective thermal conductivity of the whole system can be obtained by:

$$
\begin{aligned}
k_{\mathrm{e}} & =\frac{1}{R_{\mathrm{t}}} \frac{L}{A}=\frac{1}{R_{\mathrm{nt}}} \frac{L}{A}+\frac{1}{R_{\mathrm{SC}}} \frac{L}{A}=\frac{1}{R_{\mathrm{nt}}} \frac{A_{\mathrm{nt}}}{A}+\frac{1}{R_{\mathrm{SC}}} \frac{L}{A_{\mathrm{SC}}} \frac{A_{\mathrm{SC}}}{A} \\
& =\frac{A_{\mathrm{nt}}}{A} k_{\mathrm{e}, \mathrm{nt}}+\left(1-\frac{A_{\mathrm{nt}}}{A}\right) k_{\mathrm{e}, \mathrm{SC}}^{+(n)}
\end{aligned}
$$

where the subscript nt represents the non-touching particle, $A$ is the total area of a representative cross section, and $A_{\mathrm{nt}}$ is an equivalent area of a cross section having the same porosity as the non-touching particles, with $0<A_{\text {nt }} / A<1$. For a unit cell consisting of non-touching particles, Hsu et al. [15] obtained the following expression for the thermal conductivity:

$$
k_{\mathrm{e}, \mathrm{nt}}=k_{\mathrm{m}}(1-\sqrt{1-\phi})+\frac{k_{m} \sqrt{1-\phi}}{1+(1 / \beta-1) \sqrt{1-\phi}}
$$

Combining eqs. (9), (11) and (12), the effective thermal conductivity can be obtained by:

$$
\begin{aligned}
k_{\mathrm{e}}^{+} & =\frac{k_{\mathrm{e}}}{k_{\mathrm{m}}}=\frac{A_{\mathrm{nt}}}{A} k_{\mathrm{e}, \mathrm{nt}}^{+}+\left(1-\frac{A_{\mathrm{nt}}}{A}\right) k_{\mathrm{e}, \mathrm{SC}}^{+(n)} \\
& =\frac{A_{\mathrm{nt}}}{A}\left[(1-\sqrt{1-\phi})+\frac{\sqrt{1-\phi}}{1+(1 / \beta-1) \sqrt{1-\phi}}\right]+\left(1-\frac{A_{\mathrm{nt}}}{A}\right) k
\end{aligned}
$$

where the superscript $n=1,2,3 \ldots k_{\mathrm{e}, \mathrm{nt}}^{+}=k_{\mathrm{e}, \mathrm{nt}} / k_{\mathrm{m}}$ and $k_{\mathrm{e}, \mathrm{SC}}^{+}$is given by eq. (9).

\section{Results and discussion}

\subsection{Effective thermal conductivity of dry sand}

There are two base types of porous materials in nature. One is the granular or particulate materials, in which the void volume may be occupied by either liquid or gaseous components. And the other is a material having a continuous solid matrix that contains pores/bubbles, which may be isolated or interconnected. To verify the model and assess the contact thermal resistance, the effective thermal conductivities of dry sand and a pseudo-porous food gel, where the expanded polystyrene (EPS) beads were suspended in the gel, have been studied $[17,18]$. Figure 5 exhibits the experimental and evaluation results of the thermal conductivities. The predictions of series model (arithmetic model) and parallel model (harmonic model) [8] are also shown in Figure 5. In this evaluation, the thermal conductivities and other parameters, which were used in the eqs. (2), (10) and (12), are listed in Table 1. From the relation curves of thermal conductivity with porosity, it can be observed that the calculated results of the fractal model agree well with the ex-

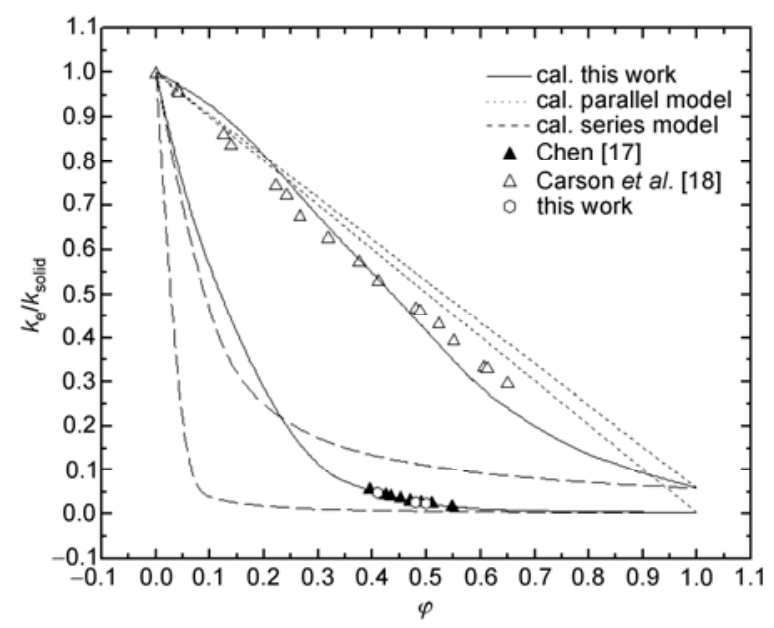

Figure 5 Experimental and predicted results of thermal conductivity for porous materials. 
Table 1 Thermal conductivities and model parameters

\begin{tabular}{ccc}
\hline \multicolumn{3}{c}{ Thermal conductivity $\left(\mathrm{W} \mathrm{m}^{-1} \mathrm{~K}^{-1}\right)$} \\
\hline Sand grain mineral & 7.5 & Chen [17] \\
Air $\left(30^{\circ} \mathrm{C}\right)$ & 0.026 & Kaye et al. $[19]$ \\
Food gel & 0.60 & Carson et al. $[18]$ \\
$2 \mathrm{~mm}$ EPS & 0.035 & Carson et al. $[18]$ \\
& Parameter & eq. (1) \\
$C$ & 3 & eq. (1) \\
$t^{+}$ & 1 & eq. (9) \\
$A_{\mathrm{nt}} / \mathrm{A}$ & 0.0001 (sand) & eq. (13) \\
\hline
\end{tabular}

perimental results.

In Figure 5, only the contact thermal resistance parameter $t^{+}(=t / L<C / L)$ is different. It is just as our expectations. Large $t^{+}$value means more solid matrix is contacted or linked. For particulate-type materials in which air (for instance) comprises a continuous phase, $t^{+}$is small in number; in most cases, it is smaller than 0.001 .

It can be known from the comparisons that the fractal model proposed in this paper can rationally describe the changing law of the sandy porous medium in thermal conductivity, and the model becomes simpler and clearer with just a few coefficients that have specific physical meanings.

\subsection{Effective thermal conductivity of porous methane hydrates}

Stern et al. [20] suggested that accurate sample characterization is critical to understanding the hydrate growth, formation, and dissociation processes, as well as to reliably interpreting physical property measurements. However, the hydrate sample formed in laboratory always mixed with pores filled with water or methane gas. In general, compaction with mechanical pressure can reduce the pores of hydrate samples, but cannot eliminate them because the pores may be not interconnected, which means the methane gas can not penetrate the hydrate shell and escape from the pores. Figure 6 shows the thermal conductivities of methane hydrate sample under different temperatures and different compaction pressures. The thermal conductivity increases with the increment of porosity, but the increment is not obvious above $10 \mathrm{MPa}$. Waite et al. [21] reported their experiment at higher radial compaction pressure (102 $\mathrm{MPa}$ ) and with modifications to their equipment to permit more uniform compaction of the hydrate sample. Their result is about $10 \%$ higher than ours. However, their sample still contained more pores than that of Rosenbaum et al. [22].

Figure 7 shows some experimental results of the thermal conductivity of porous methane hydrate and the relation curves of thermal conductivity with porosity for 3 given thermal conductivities of zero-porosity methane hydrate. The porosity was calculated by the density of the porous methane hydrate sample and the density of zero-porosity methane hydrate is assumed to be $910 \mathrm{~kg} \mathrm{~m}^{-3}$. The thermal

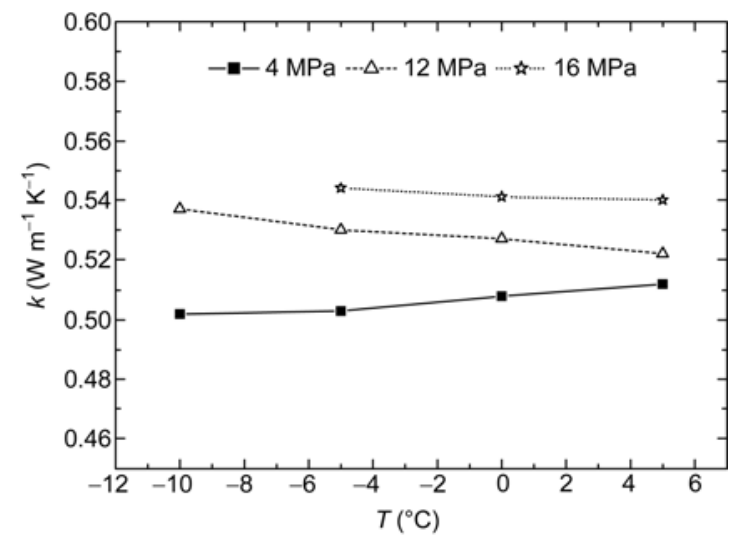

Figure 6 The influences of temperature and compaction pressure on thermal conductivity of methane hydrate sample.

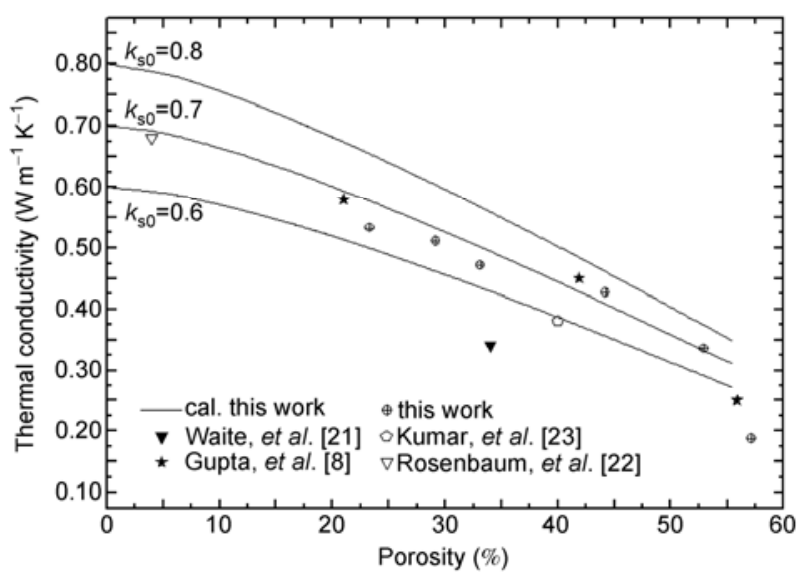

Figure 7 Comparison between estimated effective thermal conductivity, literature values, and predictions of mixing models.

conductivities of zero-porosity methane hydrate $\left(\mathrm{k}_{\mathrm{s} 0}\right)$ are assumed to be $0.6,0.7$ and $0.8 \mathrm{~W} \mathrm{~m}^{-1} \mathrm{~K}^{-1}$ and the thermal conductivity of methane gas is $0.037 \mathrm{~W} \mathrm{~m}^{-1} \mathrm{~K}^{-1}$.

Here, the contact resistance parameter $t^{+}$is 0.3 . Hence, hydrate is like foam, which is filled with free methane gas, rather than unconsolidated particles. As shown in Figure 7, the influence of porosity on the effective thermal conductivity is obvious. It exhibits a decrease in the thermal conductivity of porous methane hydrate sample with increment of porosity. The porosity of 0.3 can reduce the thermal conductivity of methane hydrate by $25 \%$. Because the quantitative values of experimental results of the thermal conductivity are much different, it is difficult to use an appropriate model to fit all experimental data. Up to now, the highest value thermal conductivity of methane hydrate, which was reported by Rosenbaum and his cooperator, was about 0.68 $\mathrm{W} \mathrm{m}{ }^{-1} \mathrm{~K}^{-1}$ [22]. Gupta et al. [8] determined the composite thermal conductivity of a porous methane hydrate sample and the value ranged between 0.25 and $0.58 \mathrm{~W} \mathrm{~m}^{-1} \mathrm{~K}^{-1}$ as a function of density and predicted nonporous methane hydrate thermal conductivity by employing some inverse 
models. In their evaluation, an optimized value is 0.70 $( \pm 0.04) \mathrm{W} \mathrm{m}^{-1} \mathrm{~K}^{-1}$. By analysis of our experimental data and simulative result, the optimized value of the thermal conductivity for zero-porosity methane hydrate sample is about $0.7 \mathrm{~W} \mathrm{~m}^{-1} \mathrm{~K}^{-1}$. If this conjecture is true, it is necessary to revaluate the economical efficiency and the safety of hydrate exploitation.

\section{Conclusions}

The effective thermal conductivities of gas-saturated porous hydrates were determined by a single-sided TPS technique and simulated by a generalized fractal model of porous media based on self-similarity. The density of porous hydrate, which was measured by the volume of the sample in the experimental system, was used to determine the porosity of methane hydrate samples. In this work, compaction with mechanical pressure can reduce the pores of hydrate samples, but can't eliminate them. The thermal conductivity increases with the increment of porosity, but the increment is not obvious at the pressure beyond $10 \mathrm{MPa}$.

In addition, a fractal model, which was based on a Sierpinski carpet model and a thermal-electrical analogy technique and one-dimensional heat flow assumption, was used to predict the effective thermal conductivity of the porous methane hydrate. Both the experimental and computational results show the effective thermal conductivity of methane hydrate decreases with the porosity increment. A porosity of 0.3 can reduce the thermal conductivity of porous methane hydrate by $25 \%$. Through analysis of the experimental data and the simulative result, the optimized estimation value of the thermal conductivity is about $0.7 \mathrm{~W} \mathrm{~m}^{-1} \mathrm{~K}^{-1}$ for the zeroporosity methane hydrate.

This work was supported by the National Basic Research Program of China (2009CB219504), the National Natural Science Foundation of China (50706056, U0933004) and Guangdong Province Science and Technology Program (2009B030600005).

1 Sloan ED. Clathrate Hydrates of Natural Gases. 2nd ed. New York: Marcel Dekker Inc., 1998

2 Huang DZ, Fan SS. Thermal conductivity of methane hydrate formed from sodium dodecyl sulfate solution. J Chem Eng Data, 2004, 49: 1479-1482

3 Li XS, Zhang Y, Li G, Chen ZY, Yan KF, Li QP. Gas hydrate equilibrium dissociation conditions in porous media using two thermodynamic approaches. J Chem Thermodyn, 2008, 40: 1464-1474
4 Tang CP, Dai XX, Du JW, Li DL, Zang XY, Yang XY, Liang DQ. Kinetic studies of gas hydrate formation with low-dosage hydrate inhibitors. Sci China Chem, 2010, 53: 2622-2627

5 Kuhs WF, Klapproth A, Gotthardt F, Techmer K, Heinrichs T. The formation of meso- and macroporous gas hydrates. Geophys Res Lett, 2000, 27: 2929-2932

6 Rosenbaum EJ. Thermal Properties and Characterization of Methane Hydrates. M.S. thesis, University of Pittsburgh. 2003

7 deMartin BJ. Laboratory Measurements of the Thermal Conductivity and Thermal Diffusivity of Methane Hydrate at Simulated in situ Conditions. M.S. Thesis, Georgia Institute of Technology, 2001

8 Gupta A, Kneafsey TJ, Moridis GJ, Seol Y, Kowalsky MB, Sloan ED Jr. Composite thermal conductivity in a large heterogeneous porous methane hydrate sample. J Phys Chem B, 2006, 110: 16384-16392

9 Thovert JF, Wary F, Adler PM. Thermal conductivity of random media and regular fractals. J Appl Phys, 1990, 68: 3872-3883

10 Adler PM. Transports in fractal porous media. J F Hydrology, 1996, 187: 195-213

11 Ma YT, Yu BM, Zhang DM, Zou MQ. A self-similarity model for effective thermal conductivity of porous media. J Phys D: Appl Phys, 2003, 36: 2157-2164

12 ZhaoYL, Guo KH, Liang DQ, Fan SS, Liu XC, Shu BF, Ge XS, Liu Y. Formation process and fractal growth model of HCFC-141b refrigerant gas hydrate. Sci China Ser B, 2002, 45: 216-224

13 Yang HJ, Li XS, Chen ZY, Yan KF, Li G., Huang NS. Study on the dissociation behavior of gas hydrate in porous sediment based on fractal theory. Acta Chimica Sinica, 2009, 67: 808-812

14 Mandelbrot BB. The Fractal Geometry of Nature. New York: WH Freeman and Company, 1982

15 Hsu CT, Cheng P, Wong KW. A lumped-parameter model for stagnant thermal conductivity of spatially periodic porous media. $J$ Heat Transfer, 1995, 117: 264-269

$16 \mathrm{Yu} \mathrm{B}$, Chen P. Fractal models for the effective thermal conductivity of bi-dispersed Porous media. J Thermophys Heat Tr, 2002, 16: 22-29

17 Chen SX. Thermal conductivity of sands. Heat Mass Transfer, 2008, 44: 1241-1246

18 Carson JK, Lovatt SJ, Tanner DJ, Cleland AC. Experimental measurements of the effective thermal conductivity of a pseudo-porous food analogue over a range of porosities and mean pore sizes. J Food Eng, 2004, 63: 87-95

19 Kaye GWC, Laby TH. Tables of Physical and Chemical Constants. 16th ed. Middlesex: National Physical Laboratory, 2007. (available at http://www.kayelaby.npl.co.uk)

20 Stern LA, Kirby SH, Circone S, Durham WB. Scanning electron microscopy investigations of laboratory-grown gas clathrate hydrates formed from melting ice, and comparison to natural hydrates. Am Mineral, 2004, 89: 1162-1175

21 Waite WF, Pinkston J, Kirby SH. Preliminary laboratory thermal conductivity measurements in pure methane hydrate and methane hydrate-sediment mixtures: A progress report. In: Proceedings of 4th International Conference on Gas Hydrates. Yokohama, Japan. 2002. 728-733

22 Rosenbaum EJ, English NJ, Johnson JK, Shaw DW, Warzinski RP. Thermal conductivity of methane hydrate from experiment and molecular simulation. J Phys Chem B, 2007, 111: 13194-13205

23 Kumar P, Turner D, Sloan ED Jr. Thermal diffusivity measurements of porous methane hydrate and hydrate-sediment mixtures. $J \mathrm{Ge}$ ophys Res, 2004, 109: B01207, doi: 10.1029/2003JB002763 